\title{
Fast Region-based Active Contour Model Driven by Local Signed Pressure Force
}

\author{
Abdallah Azizi ${ }^{*}$ and Kaouther Elkourd ${ }^{+}$ \\ * Department of Electrical Engineering, University of Mohamed Khider, B.P 145 RP, Biskra, ALGERIA \\ + Department of Physics, Preparatory School for Science \& Techniques, B.P 474 Martyrs' Square, Algiers, ALGERIA
}

Received 4th Aug 2015; accepted 14th Jan 2016

\begin{abstract}
Intensity inhomogeneity is a well-known problem in image segmentation. In this paper, we present a new region-based active contour model for image segmentation which can handle intensity inhomogeneity problem. This model introduced a new region-based signed pressure force (SPF) function, which uses the local mean values provided by the local binary fitting (LBF) model. In addition, the proposed model utilizes a new regularization operation such as morphological opening and closing to regularize the level set function in the evolution process. Experimental results on synthetic and real images show that the proposed model gives satisfactory segmentation results as well as less sensitivity to the initial contour location and less time consuming compared with the related methods.
\end{abstract}

Key Words: Active contour models, Image segmentation, Intensity inhomogeneity, Local image information, Signed pressure force function.

\section{Introduction}

Image segmentation is a fundamental procedure in the field of computer vision and image processing. Different methods have been proposed for image segmentation including the Active Contour Models (ACM) [1]. The fundamental idea of ACM is to evolve a curve under some constraints from a given image to reach the boundaries of the desired objects, by minimizing energy functional. According to the energy, ACM are classified into two main categories: edge-based models [1-4] and region-based models [5-8].

Edge-based models rely on the image gradient information to stop the evolving curve on the desired object boundaries. Geodesic Active Contour (GAC) [2] is one of the well-known models in this category. Although GAC has been successfully applied for images with high variation in gradient at the object's boundaries, it meets difficulty when dealing with the objects having blurred or discrete boundaries and it hardly detects the object corrupted by noise [9].

Unlike to edge-based models, the region-based models do not utilize the image gradient; they utilize image statistics inside and outside the curve to control the evolution with better performance including weak edges and noise. One of the famous region-based models is the Chan and Vese (C-V) model [5] which is a simplified Mumford-Shah model [10]. The C-V model utilizes the global information of homogeneous

Correspondence to: <aziziabdallah87@yahoo.com>

Recommended for acceptance by Xiaoyi Jiang

http://dx.doi.org/10.5565/rev/elcvia.794

ELCVIA ISSN: 1577-5097

Published by Computer Vision Center / Universitat Autonoma de Barcelona, Barcelona, Spain 
regions. Thus, it has good segmentation result for the objects with weak or discrete boundaries but often has erroneous segmentation for images with intensity inhomogeneity [11].

In order to deal with intensity inhomogeneity, Li et al [12] proposed the Local Binary Fitting (LBF) model by using local regions information instead of global ones. However, it is sensitive to initial contour and is easy to trap into local minimum, which holds back its practical applications $[13,14]$.

To ameliorate the robustness to contour initialization, Zhang et al [6] proposed a hybrid model combining the local and global intensity information, which utilizes a region-based Signed Pressure Force (SPF) function to update curve evolution and a special processing named Selective Binary and Gaussian Filtering Regularized Level Set (SBGFRLS) method to regularize the Level set function. This model is robust and simple to implement, but it is found to be slow and parameter dependable [7].

An Online Region-based Active Contour Model (ORACM) using a new level set formulation was proposed in [7], which present the advantage by providing less time without changing segmentation accuracy and parameter free. Different to the ACM with SBGFRLS, ORACM uses a simple and efficient level set updating formulation by using directly current level set function instead of the curvature approximation and a simple morphological opening and closing processes instead of Gaussian smoothing to smooth the level set function. However, this model is hard to deal with the images having inhomogeneous intensity [7].

Inspired by the work in [7] and [12], we propose a Fast Local Region-based ACM which deals well under the intensity inhomogeneity problem. It is implemented by introducing a new SPF function that utilizes the local image information provided by the LBF model in the level set updating formulation of the ORACM model. Similar to ORACM model, the proposed model uses a simple morphological opening and closing regularization operation to regularize the level set function, and in addition to ORACM model advantages, the proposed model can effectively segment images with intensity inhomogeneity. Experiments on some synthetic and real images show desirable segmentation results of our model. Moreover, comparisons with the related models also show that our model is less sensitive to initial contour location and more computationally efficient.

This paper is organized as follow: In section 2, we review some ACM and their limitations. Section 3 describes the proposed model. Experimental results are shown in section 4. Finally, Section 5 concludes the paper.

\section{Related Methods}

\subsection{The GAC model}

Let $I$ : $\Omega \rightarrow R$ be a given image, $\Omega$ be the image domain and $C$ be a closed contour. The GAC model [2] is formulated by minimizing the following function:

$$
E^{G A C}(C(q))=\int_{0}^{1} g(\nabla I(C(q)))\left|C^{\prime}(q)\right| d q,
$$

where $g$ is an Edge Stopping Function (ESF) defined by:

$$
g(|\nabla I|)=\frac{1}{1+\left|\nabla G_{\sigma} * I\right|^{2}},
$$

where $G_{\sigma}$ is a Gaussian Kernel with standard deviation $\sigma$.

Using calculus of variation [15], the Euler-Lagrange equation of (1) is:

$$
C_{t}=g(\nabla I \mid)(k+\alpha) \vec{N}-(\nabla g \cdot \vec{N}) \vec{N},
$$

where $k$ is the curvature of the contour, $\vec{N}$ is the inward normal to the curve and $\alpha$ is a constant which is added to increase the propagation speed. By using the level set method [16], we can obtain the following level set formulation: 


$$
\frac{\partial \phi}{\partial t}=g\left(\operatorname{div}\left(\frac{\nabla \phi}{|\nabla \phi|}\right)+\alpha\right)(|\nabla \phi|)+\nabla g \cdot \nabla \phi
$$

Because of the GAC model relies on the ESF $g$ to stop the curve evolution, the model can detect only objects with edges defined by gradient [17].

\subsection{The C-V model}

Chan and Vese [5] proposed an active contour model does not rely on the ESF during curve evolution. This region-based active contour is a special case of Mumford-shah problem [10], which uses the intensity information of different regions to minimize the energy functional defined as follow:

$$
\left.E^{C V}\left(C_{1}, C_{2}, C\right)=\mu \text {.length }(C)+v \text {.area (inside }(C)\right)+\lambda_{1} \int_{\text {inside }(C)}\left|I(x)-C_{1}\right|^{2} d x+\lambda_{2} \int_{\text {ousside }(C)}\left|I(x)-C_{2}\right|^{2} d x,
$$

where $\mu, v \geq 0$ and $\lambda_{1}, \lambda_{2}>0$ are constants. $C_{1}$ and $C_{2}$ are the average intensities of $I$ inside and outside the curve, respectively. Both length of the curve and the area of the region inside $C$, regularize the curve. By the use of the level set method [16], the energy functional in (5) can be reworded as:

$$
E^{C V}\left(C_{1}, C_{2}, \phi\right)=\mu \int_{\Omega} \delta(\phi)|\nabla \phi(x)| d x+v \int_{\Omega} H(\phi(x)) d x+\lambda_{1} \int_{\text {inside }(C)}\left|I(x)-C_{1}\right|^{2} H(\phi(x)) d x+\lambda_{2} \int_{\text {ousside }(C)}\left|I(x)-C_{2}\right|^{2}(1-H(\phi(x))) d x,
$$

where $H(\phi)$ and $\delta(\phi)$ are the Heaviside function and the Dirac function, respectively.

Using the Euler-Lagrange equation to solve the minimization problem of (6), the corresponding level set formulation can be expressed as:

$$
\frac{\partial \phi}{\partial t}=\delta(\phi)\left[-\lambda_{1}\left(I-C_{1}\right)^{2}+\lambda_{2}\left(I-C_{2}\right)^{2}+\mu \operatorname{div}\left(\frac{\nabla \phi}{\nabla \phi}\right)-v\right],
$$

where $C_{1}$ and $C_{2}$ can be expressed as follow:

$$
\left\{\begin{array}{c}
C_{1}(\phi)=\frac{\int_{\Omega} I(x) \cdot H(\phi) d x}{\int_{\Omega} H(\phi) d x} \\
C_{2}(\phi)=\frac{\int_{\Omega} I(x) \cdot(1-H(\phi)) d x}{\int_{\Omega}(1-H(\phi)) d x} .
\end{array}\right.
$$

The C-V model has good performance when segmenting images with weak edges and noise. Thus, the use of global information of the image leads to inaccurate segmentation results for images with intensity inhomogeneity.

\subsection{The ACM with SBGFRLS model}

Zhang et al. proposed a region-based GAC [6] which incorporates the GAC and C-V model by constructing a SPF function replacing the ESF of the GAC model. The SPF function is defined as follow:

$$
\operatorname{SPF}(I(x))=\frac{I(x)-\frac{C_{1}+C_{2}}{2}}{\max \left(\mid I(x)-\frac{C_{1}+C_{2}}{2}\right)},
$$


where $C_{1}$ and $C_{2}$ are defined in (8). The SPF function modulates the signs of the pressure force inside and outside the region of interest so that the curve shrinks when outside the object, or expands when inside the object. The level set formulation of this model is given as:

$$
\frac{\partial \phi}{\partial t}=S P F(I(x)) \cdot \alpha \cdot|\nabla \phi|
$$

The use of the SPF function makes the model controls the direction of the evolution and to stop the evolving curve at weak or blurred edges. Moreover, the level set function of this model is regularized by the selective binary and Gaussian filtering SBGFRLS which reduces the computational coast of the reinitialization step which in turn makes it more efficient than the traditional level set methods [8]. However, the ACM with SBGFRLS has two major disadvantages: the parameter $\alpha$ must be tuned according to images which make the model parameter dependable. The second disadvantage is slowness causes by the gradient of the level set function [7].

\subsection{The ORACM model}

ORACM [7] is a region based active contour which covers the drawbacks provided by ACM with SBGFRLS; it necessitates no parameter and takes less time to detect objects boundaries. Unlike the ACM with SBGFRLS, ORACM uses a simple and efficient level set updating formulation given as follow:

$$
\frac{\partial \phi}{\partial x}=H(S P F(I(x))) \cdot \phi(x)
$$

where $H($.) is the Heaviside function and $\operatorname{SPF}($.$) is the signed pressure force function defined in (9).$ Comparing with the level set formulation of the ACM with SBGFRLS, ORACM does not need parameters to be tuned according to input images, which make it parameter free. The directly use of current level set function instead of its curvature approximation reduces the calculation time. In addition, ORACM uses a simple morphological opening and closing processes instead of Gaussian smoothing to smooth updated level set.

In spite of having a considerable amelioration from the perspective of speed and parameter dependability, using global information in the SPF function tends to erroneous segmentation results with images having inhomogeneity of intensity.

\subsection{The LBF model}

In order to overcome the problem of intensity inhomogeneity, Li et al. proposed the LBF model [12]; by using two smooth functions $f_{l}(x)$ and $f_{2}(x)$ that approximate the local image intensity inside and outside the curve, respectively. The energy functional is defined as follow:

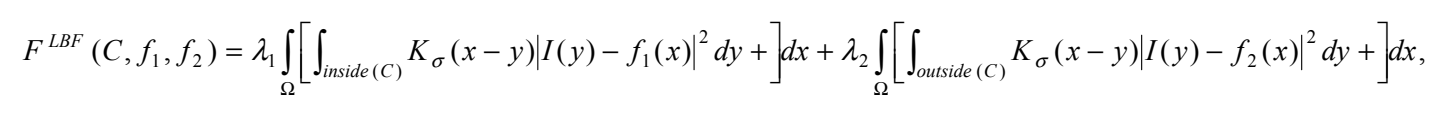

where $\lambda_{1}$ and $\lambda_{2}$ are positive constants and $K_{\sigma}$ is a Gaussian kernel with standard deviation $\sigma$.

Using level set function $\phi$ to represent the curve $C$ and minimizing the energy functional $F^{L B F}$ with respect to $\phi$, give the following gradient descent flow:

$$
\frac{\partial \phi}{\partial t}=-\delta(\phi)\left[\lambda_{1} \int_{\Omega} K_{\sigma}(y-x)\left|I(x)-f_{1}(y)\right|^{2} d y-\lambda_{2} \int_{\Omega} K_{\sigma}(y-x)\left|I(x)-f_{2}(y)\right|^{2} d y\right]
$$

where $f_{1}(x)$ and $f_{2}(x)$ are defined as follow: 


$$
\left\{\begin{aligned}
f_{1}(x) & =\frac{\int_{\Omega} K_{\sigma} *[I(x) H(\phi)]}{\int_{\Omega} K_{\sigma} * H(\phi)} \\
f_{2}(x) & =\frac{\int_{\Omega} K_{\sigma} *[I(x)(1-H(\phi))]}{\int_{\Omega} K_{\sigma} *(1-H(\phi))} .
\end{aligned}\right.
$$

Because of using local region information, specifically local intensity mean, the LBF model is able to provide desirable segmentation results even in the presence of intensity inhomogeneity. However, it is sensitive to initialization to some extent and easy to fall into a local minimum [13].

\section{The Proposed Method}

Based on the level set updating formulation of the ORACM model, the proposed model is constructed by replacing the SPF function with a new SPF function, taking into account the local intensity information.

\subsection{Local SPF function}

In our model, we introduce a new SPF function based on the local properties of the image by replacing the global mean values inside and outside the curve with the local mean values provided by the LBF model. The new SPF function is called Local Signed Pressure Force (LSPF) function which is constructed as follow:

$$
\operatorname{LSPF}(I(x))=\frac{I(x)-\frac{f_{1}+f_{2}}{2}}{\max \left(\mid(x)-\frac{f_{1}+f_{2}}{2}\right)},
$$

where $f_{1}$ and $f_{2}$ are defined in (14).

Similar to the SPF defined in [6], the sign and value of LSPF ranges in $[-1,1]$ so that, it adjusts the signs of the pressure force inside and outside the region of interest so that the curve shrinks when outside the object and expands when inside the object; the only difference is that the LSPF is constructed using local mean values inside and outside the curve which help the model to work well with the intensity inhomogeneous regions, where the LSPF can be minimized when the curve is exactly on the region boundary; the local mean intensities which are computed using the Gaussian kernel as in (14), lead to assign different signs for both inside and outside region presenting inhomogeneous intensity.

In addition, the proposed model is a generalization of the ORACM model, and it is degenerated when the Kernel function in (14) is an averaging filter and the size of its window is infinity; the functions $f_{1}$ and $f_{2}$ become representing average intensities inside and outside the curve $C_{1}$ and $C_{2}$, respectively.

\subsection{Implementation}

In practice, the Heaviside function $H($.$) in (14) is approximated by a smoothed functional H_{\epsilon}($.) defined, as proposed in [12], by:

$$
H_{\varepsilon}(x)=\frac{1}{2}\left[1+\frac{2}{\pi} \arctan \left(\frac{x}{\varepsilon}\right)\right] .
$$

Substituting the SPF function in (11) with the LSPF function defined in (15), we obtain the level set updating formulation of the proposed model as follow:

$$
\frac{\partial \phi}{\partial x}=H(L S P F(I(x))) \cdot \phi(x)
$$


Similar to ORACM model, the proposed model uses a simple morphological opening and closing processes to smooth updated level set. These two operations performed sequentially are commonly used in computer vision and image processing for noise removal and smoothing [7].

Finally, the different steps of the proposed algorithm are as follow:

1. Initialization of the level set function $\phi$ to be a binary function as follow:

$$
\phi_{0}(x, y)=\left\{\begin{array}{cl}
-d & (x ; y) \in \Omega_{0}-\partial \Omega_{0} \\
0 & (x ; y) \in \partial \Omega_{0} \\
+d & (x ; y) \in \Omega-\partial \Omega_{0}
\end{array}\right.
$$

where $d>0$ is a constant, $\Omega_{0}$ is a subset in the image domain $\Omega$, and $\partial \Omega_{0}$ be all the points in the boundaries of $\Omega_{0}$.

2. Compute the local mean values $f_{1}(x)$ and $f_{2}(x)$ according to (14).

3. Calculate the LSPF function $\operatorname{LSPF}(I(x))$ using (15).

4. Evolve the level set function according to (17).

5. Check whether the evolution is stationary, stop, else go to step 2.

6. Regularize the level set function using a simple morphological opening and closing processes.

\section{$4 \quad$ Results}

In this section, we apply and compare the proposed model with the different models using both synthetic and real images. All models are implemented using Matlab 7.0 in Windows 7; on $3.3 \mathrm{GHz}$ Intel core i3 PC with 4GB of RAM. Unless otherwise specified, the parameters are described in Table 1.

Fig. 1 shows the segmentation results of the related methods and the proposed method on a synthetic image with two objects with blurred boundaries. From the first row, we can observe that the right segmentation results cannot be obtained from the GAC model which uses edge information that causes the curve to pass over real boundaries, while the other models using region information, including the proposed one, can satisfactorily segment the two objects. Furthermore, the iterations and CPU time are listed in Table 2. It can be observed that both ORACM model and the proposed model are much faster than the related models. Accordingly, the proposed model is more efficient.

The second row of Fig. 1 demonstrates the segmentation results with tuned parameters of the related methods as follow: GAC: $\alpha=0.4, \mathrm{C}-\mathrm{V}: \mu=0.8$, SBGFRLS: $\alpha=10$ and LBF: $\lambda_{1}=1.5$. As shown, they cannot detect the boundaries of the two objects after the same number of iterations mentioned in Table 2 . While ORACM model and the proposed model, without the need of any parameter, they correctly detect the boundaries of the objects after only 5 iterations. This efficiency by the proposed method caused by using the simple level set updating formulation defined in (13).

Table 1: Description of the parameters used in the study.

\begin{tabular}{|l|l|}
\hline \multicolumn{1}{|c|}{ Parameters } & \multicolumn{1}{c|}{ Description } \\
\hline$d$ & To initialize the level set function, $d>0$ is a constant. \\
\hline$\sigma$ & $\begin{array}{l}\text { Scale parameter in Gaussian kernel (LBF and our model: } \sigma \text { determined according to } \\
\text { images). }\end{array}$ \\
\hline$\lambda_{1} / \lambda_{2}$ & Inner/ outer weight of curve $C\left(\mathrm{C}-\mathrm{V}\right.$ and LBF: $\left.\lambda_{l}=\lambda_{2}=1\right)$. \\
\hline$\Delta t$ & Time step (LBF, GAC: $\Delta t=0.1 ; \mathrm{C}-\mathrm{V}, \mathrm{SBGFRLS}$, ORACM and our model: $\Delta t=1)$. \\
\hline$\alpha$ & Balloon force $(G A C: \alpha=0.8 ;$ SBGFRLS: $\alpha=25)$. \\
\hline$\varepsilon$ & The parameter of smoothed Heaviside function (LBF: $\varepsilon=1 ;$ our model: $\varepsilon=1.5)$. \\
\hline$\mu, v$ & Regularization parameters of curve $C(\mathrm{C}-\mathrm{V}: \mu=0.2, v=0)$. \\
\hline
\end{tabular}



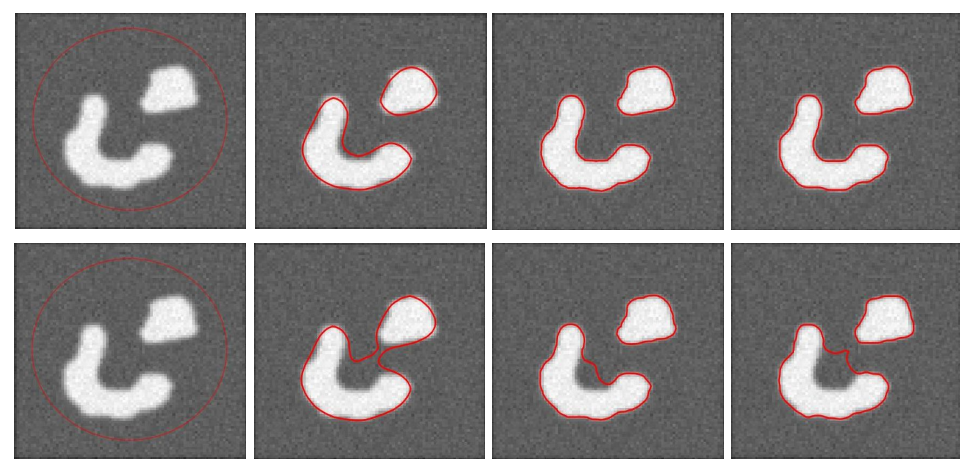

(a)

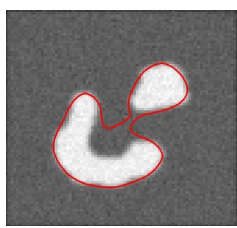

(b)

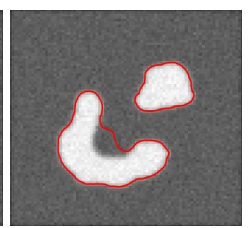

(c)
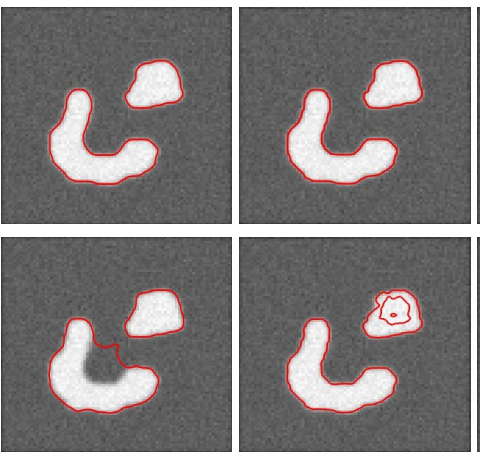

(d)

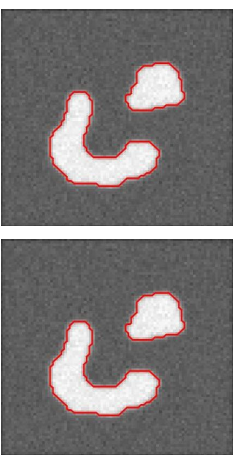

(f)

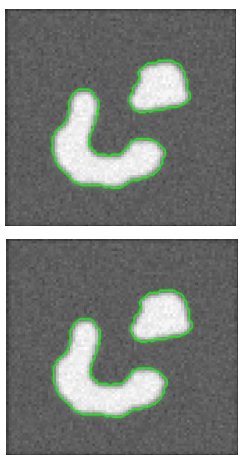

$(\mathrm{g})$

Fig. 1: Segmentation results on a synthetic image: (a) initial contour. Segmentation result by: (b) the GAC model, (c) the C-V model (d) the ACM with SBGFRLS model (e) the LBF model (f) the ORACM model (g) the proposed model. The parameter $\sigma=30$.

Table 2: Iterations and CPU time needed by the methods compared when segmenting the image in Fig. 1.

\begin{tabular}{|l|c|c|}
\hline \multicolumn{1}{|c|}{ Method } & Iterations & CPU time (s) \\
\hline The GAC model & 2000 & 72.44 \\
\hline The C-V model & 80 & 5.22 \\
\hline The ACM with SBGFRLS model & 25 & 3.30 \\
\hline The LBF model & 10 & 2.33 \\
\hline The ORACM model & 5 & 1.71 \\
\hline The Proposed model & 5 & 1.71 \\
\hline
\end{tabular}

To demonstrate the local segmentation property of the proposed model, in Fig. 2 we compare our method with the related methods on a real microscope cell image where the objects boundaries are distinctive while interior intensities are not homogeneous. It can be observed that the GAC model based on edge information can extract the objects boundaries, while other related methods which are based on region information fail to extract them. Compared to the related models based on region information, the proposed model achieve better results, because our model uses local signed pressure force in its level set formulation that has the property of local segmentation.

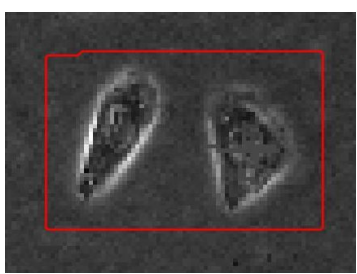

(a)

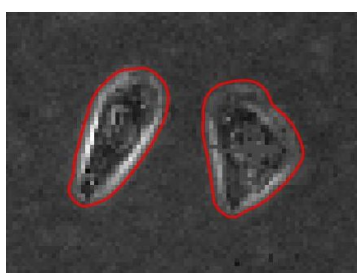

(b)

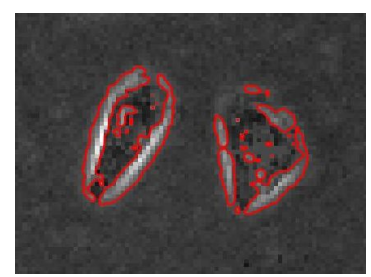

(c)

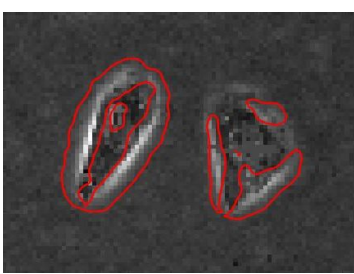

(d)

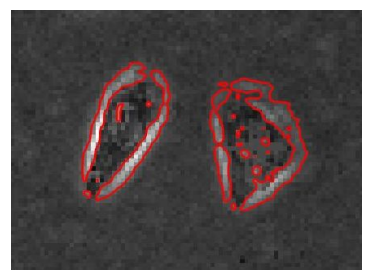

(e)

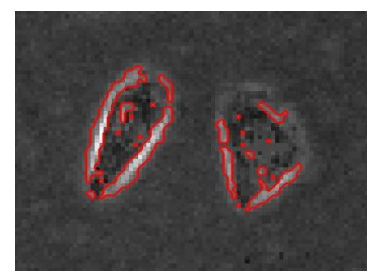

(f)

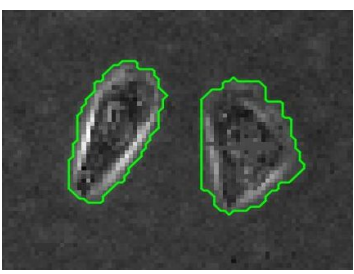

$(\mathrm{g})$

Fig. 2: Applications to a microscope cell image (downloaded from [4]): (a) initial contour. Segmentation result by: (b) the GAC model, (c) the C-V model (d) the ACM with SBGFRLS (e) the LBF model (f) the ORACM model (g) the proposed model. The parameter $\sigma=4$. 
Fig. 3 shows a segmentation result comparison with the related methods on image with intensity inhomogeneity. It can be seen that the methods using global region information (C-V, SBGFRLS and ORACM) cannot segment well when image has intensity inhomogeneous region in it, while the LBF model and the proposed method that uses local region information, could accurately segment intensity inhomogeneous region. For the GAC which based on edge information where no global constraints are placed on the image, this method can segment intensity inhomogeneity but suffer from the leakage problem as shown in Fig. 3(b).

In the next experiments, we will focus on comparisons between the LBF model and the proposed model on intensity inhomogeneity problem from different sides.

In Fig. 4, we apply the LBF model and the proposed model to a synthetic image with intensity inhomogeneity, the first row shows various initial contours, the second and the third rows show the segmentation results by the LBF model and the proposed model, respectively. From some initial contours, as in columns 1 and 5, the LBF model can segment well intensity inhomogeneity image, while giving bad segmentation results for other initial contours. Otherwise, the proposed model is much more robust to initial contour location and can achieve good segmentation results for all initial contours.

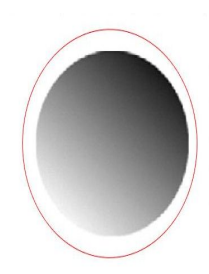

(a)

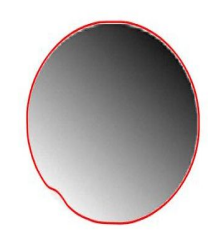

(b)

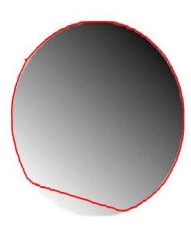

(c)

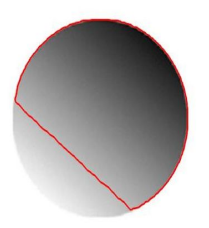

(d)

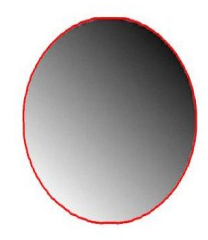

(e)

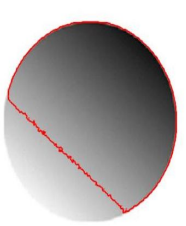

(f)

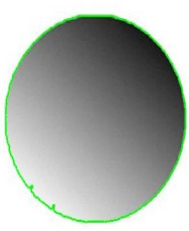

(g)

Fig. 3: Comparison of segmentation results using synthetic image with intensity inhomogeneity (downloaded from [15]): (a) initial contour. Segmentation result by: (b) the GAC model, (c) the C-V model (d) the ACM with SBGFRLS (e) the LBF model (f) the ORACM model (g) the proposed model. The parameter $\sigma=2$.
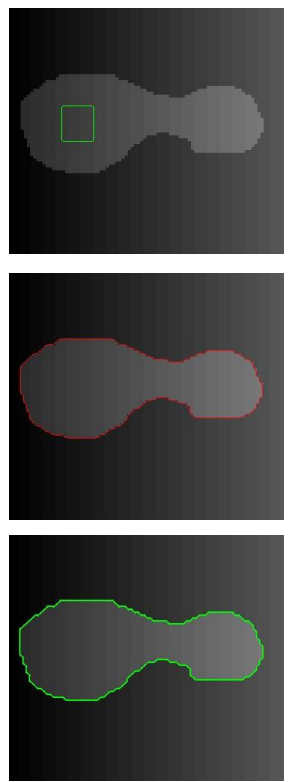
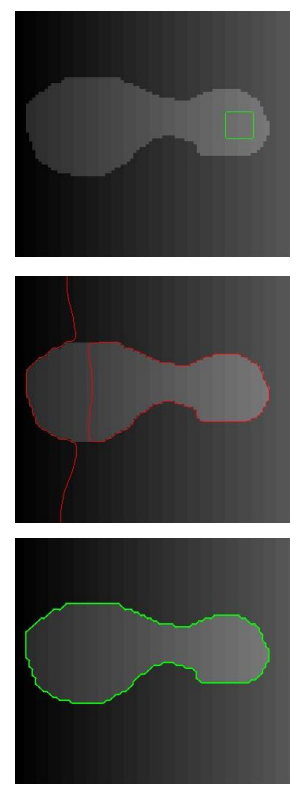
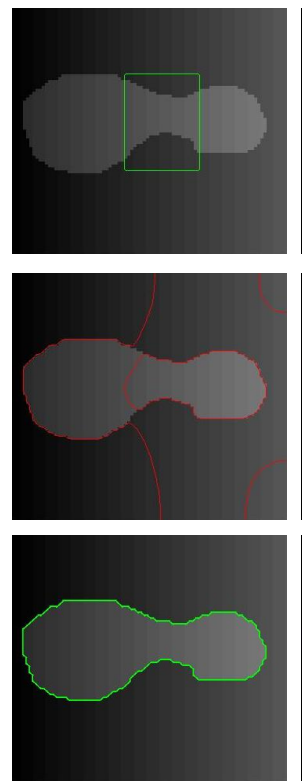
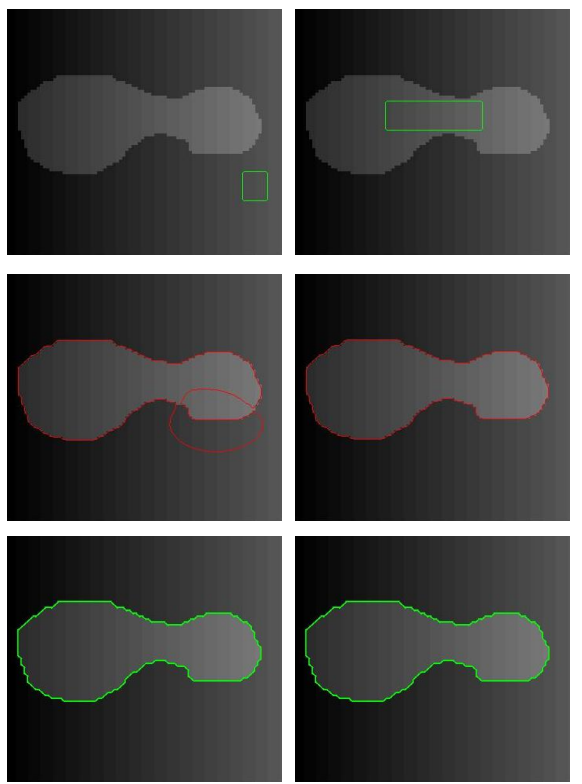

Fig. 4: Segmentation results on the synthetic image with intensity inhomogeneity. Row1: initial contours. Row2: results of the LBF model. Row3: results of the proposed model. The parameter $\sigma=5$. 
In order to compare the LBF model and the proposed model, we use other synthetic images in Fig. 5 and Fig. 6.

Fig 5(a) shows the original image with initial contour. The results of the LBF model and the proposed model are shown in Fig. 5(b) and 5(d), respectively. It is obvious that the proposed model attains better segmentation precision; it separates the boundary of each finger of the palm and reflects its shape (as can be seen clearly in the zoomed view of Fig. 5(e) better than the LBF model (zoomed up in Fig. 5(c)).

Fig .6 shows the segmentation results of another synthetic image with intensity inhomogeneity by the LBF model and the proposed model, which are shown in Fig. 6(b) and 6(c) respectively, while Fig 6(a) shows the original image with initial contour. From the results, it is clear that the LBF model fails to get the correct segmentation result and traps into local minimum, while the proposed model gets right segmentation result.

To furthermore compare the performance of the proposed model and the LBF model, on images whose intensity inhomogeneity has different strength. Fig. 7 shows the segmentation results provided by the LBF model (first row) and the proposed model (second row), on five synthetic images with different intensity inhomogeneity. Clearly, the proposed model can segment the object with different strength of intensity inhomogeneity, while the LBF model fails to segment the object when the strength of intensity inhomogeneity is strong (last two Columns).

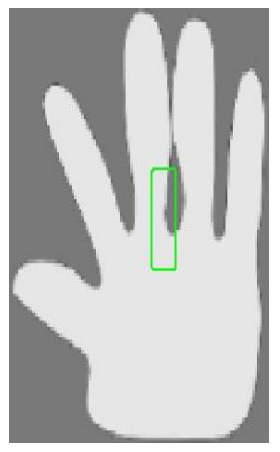

(a)

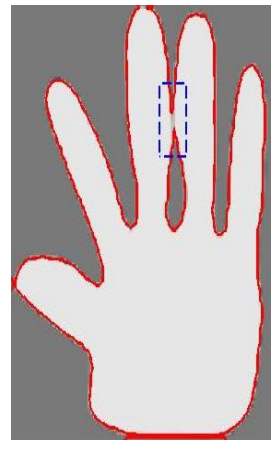

(b)

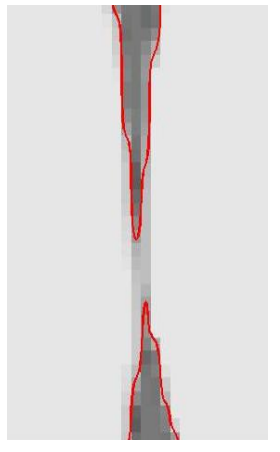

(c)

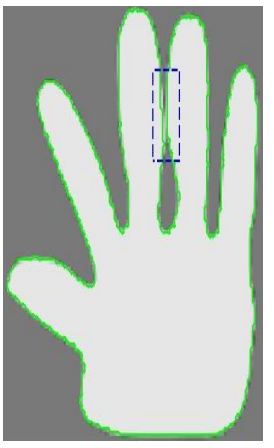

(d)

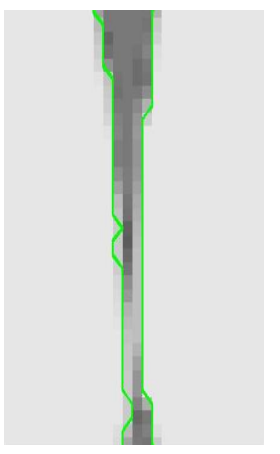

(e)

Fig. 5: Segmentation results on a hand phantom (downloaded from [6]) using the LBF and the proposed model: (a) initial contour, (b) segmentation result by the LBF model, (c) zoomed view of the narrow, blue rectangle in (b), (d) segmentation result by our method, and (e) zoomed view of the narrow, blue rectangle in (d). The parameter $\sigma=3$.

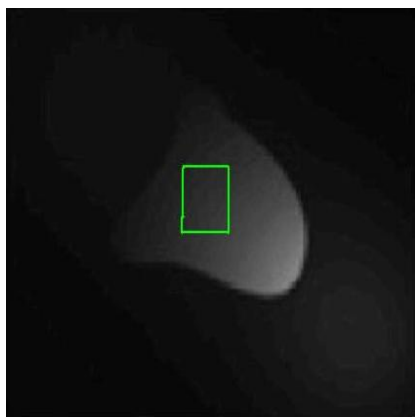

(a)

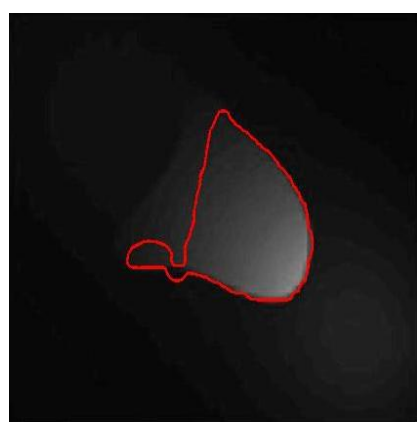

(b)

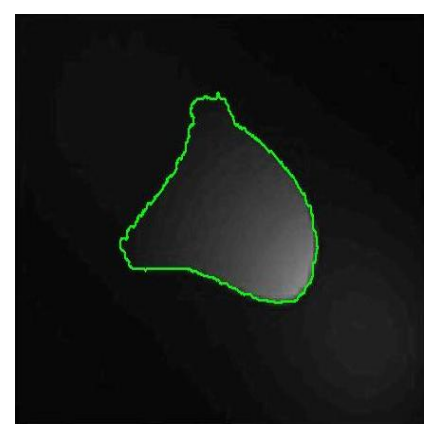

(c)

Fig. 6: Segmentation results of an inhomogeneous image (downloaded from [18]). (a) The original image with initial contour, (b) the final contour with the LBF model and (c) the final contour with the proposed model. The parameter $\sigma=30$. 

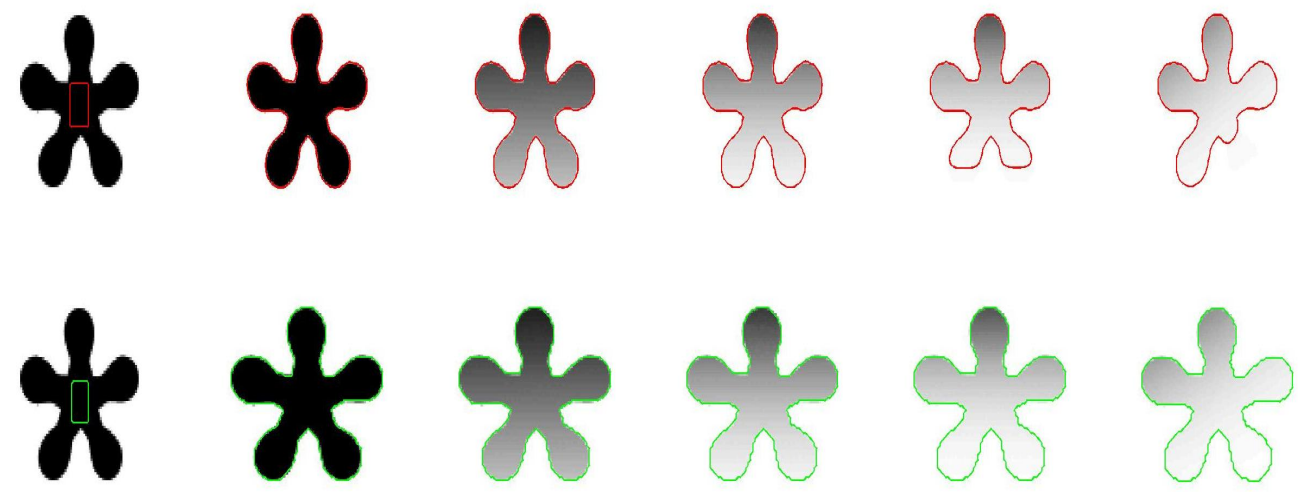

Fig. 7: Segmentation results on a synthetic image where the strength of intensity inhomogeneity is gradually increased from left to right (downloaded from [19]). Row1: results of the LBF model. Row2: results of the proposed model. The parameter $\sigma=2$.

To quantitatively validate the segmentation performance of the proposed model from Fig. 7, the Jaccard Similarity $(J S)$ index is used. The $J S$ index between the segmented object region Rs and the real object region Ro is calculated as $J S(R s, R o)=|R s \cap R o| / R s \cup R o \mid$. Clearly, when Rs is more similar to Ro, the $J S$ value is close to 1 . Fig. 8 shows that the JS values obtained by our method change in a small range for intensity inhomogeneity with different strength, while the LBF model, when the strength of intensity inhomogeneity is strong (last two columns of Fig. 7) the segmentation accuracy of this method decreases strictly. These results illustrate the robustness of the proposed model to image intensity inhomogeneity.

In Fig. 9, we apply the proposed model to segment typical medical images with different modalities and compare it to LBF model. The segmentation results provided by the LBF model are in the upper row, while the lower row presents our results. The first two columns show the results of two X-ray images of blood vessels. It can be seen that all models give satisfying segmentation results because of the use of the image local region information, which can better separate the object from background. The last three columns, from left to right, show the segmentation results of a CT image of heart and two MRI brain images with intensity inhomogeneity. It can be seen that the LBF model fails to distinguish between the intensity between the object and its background and lead to inaccurate segmentation result. While the proposed model gives much better segmentation results. These results represent the abilities of the proposed model to deal with intensity inhomogeneity and complex background.

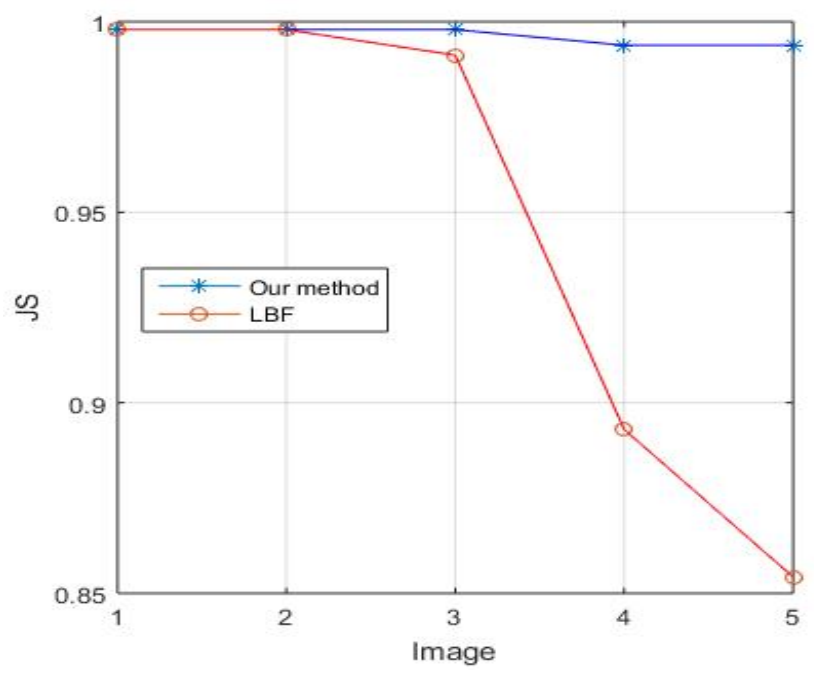

Fig. 8: The corresponding JS values yielded by the LBF model and the proposed model on the five images with different intensity inhomogeneity. 

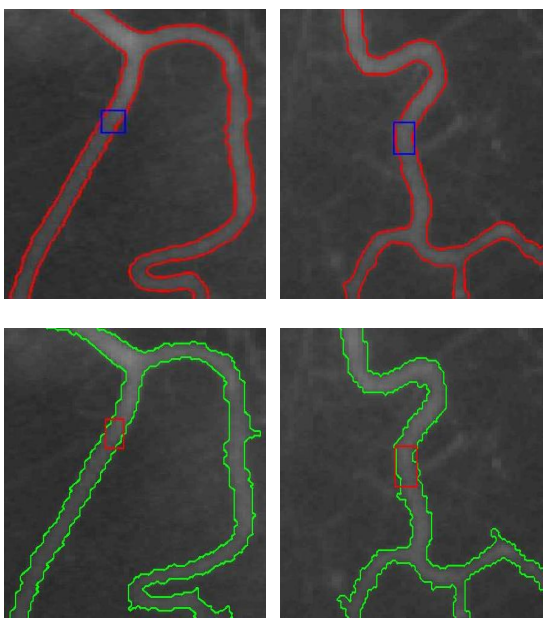

(a)

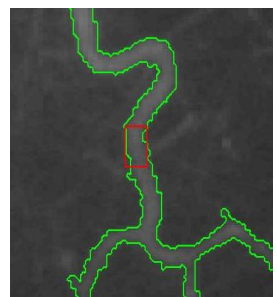

(b)
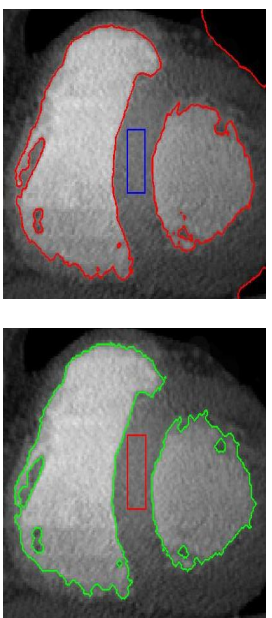

(c)
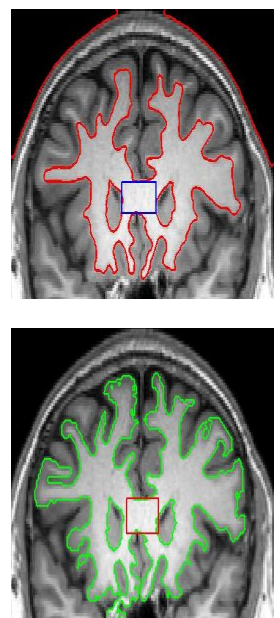

(d)
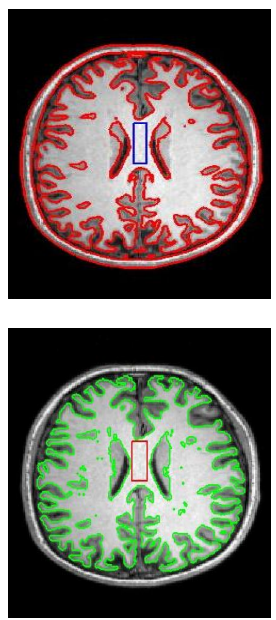

(e)

Fig. 9: Comparison of segmentation results of the LBF model (top row) and the proposed model (bottom row) on several medical images (a, b, d and e: downloaded from [19], while c: from [20]).

Furthermore, the iterations and CPU time of segmenting the images in Fig. 9, moreover the values of the parameter $\sigma$ are listed in Table 3 for the LBF model and the proposed model. It can be observed that the number of iterations and the required CPU time of the proposed model are much less than those of the LBF model. Accordingly, the proposed model is much faster than the LBF model.

To compare the performance between the LBF model and the proposed model on noisy image, we add the salt and pepper noise with various levels on the synthetic image of Fig. 4, and the segmentation results are shown in Fig. 10; the first column shows the original image with initial contour, the second, third and fourth Columns show the segmentation results on image added noise with densities: $0.05,0.1$ and 0.2 respectively. From the results, it is obvious that the proposed model has higher anti-noise than the LBF model; this performance is because of using the opening and closing morphological operations respectively to remove small objects and to smooth the active contour.

Finally, Fig. 11 shows the influence of the parameter $\sigma$ on the segmentation results of our model for an MR image of bladder with intensity inhomogeneity. The initial contour is shown in Fig. 11(a) and the result for the parameter $\sigma=8$ and $\sigma=30$ are shown in Fig. 11(b) and (c), respectively. It is clear that the corresponding result with $\sigma=8$ is more desirable, where the bladder and all small structures surrounding it are segmented. While some part of the bladder and these structures are missed in the segmentation result using $\sigma=30$, which is similar to the result of the ORACM model, shown in Fig. 10(d). As an advantage of the proposed method from these results, that it allows the choice of the scale parameter $\sigma$ to exploit intensity information in regions of different scales, so that, when the intensity inhomogeneity is severe, the accuracy of segmentation relies on the local SPF, in such case, we choose small $\sigma$, while in smooth regions, a bigger $\sigma$ is chosen; the local SPF becomes degenerating the global SPF so that the contour is attracted to the object boundary quickly.

Table 3: Iterations, CPU time and values of $\sigma$ needed when segmenting the images in Fig. 9.

\begin{tabular}{|c|c|c|c|c|c|c|}
\hline Method & \multicolumn{3}{|c|}{ The LBF model } & \multicolumn{3}{c|}{ The proposed model } \\
\hline & Parameter $\sigma$ & iterations & CPU time (s) & Parameter $\sigma$ & iterations & CPU time (s) \\
\hline Fig. 9(a) & 4 & 160 & 3.43 & 7 & 3 & 0.55 \\
\hline Fig. 9(b) & 4 & 120 & 2.04 & 10 & 11 & 1.55 \\
\hline Fig. 9(c) & 10 & 300 & 10.06 & 10 & 10 & 1.40 \\
\hline Fig. 9(d) & 3 & 60 & 5.06 & 10 & 4 & 1.01 \\
\hline Fig. 9(e) & 5 & 100 & 10.08 & 25 & 4 & 1.39 \\
\hline
\end{tabular}



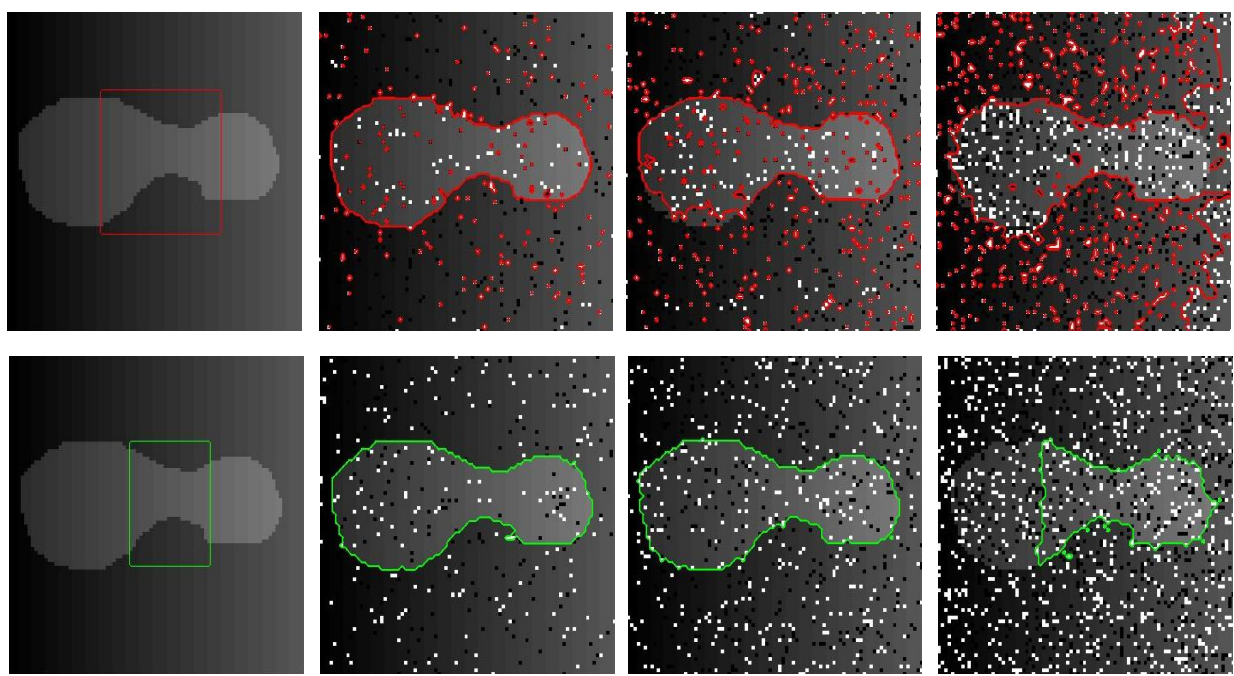

Fig. 10: Comparisons of the segmentation results with salt and pepper noise by the LBF model and the proposed model on Row1 and Row2, respectively. Column1: the original image with initial contour; Columns 2, 3 and 4 added the salt and pepper noise with densities $0.05,0.1$ and 0.2 , respectively. The parameter $\sigma=10$.

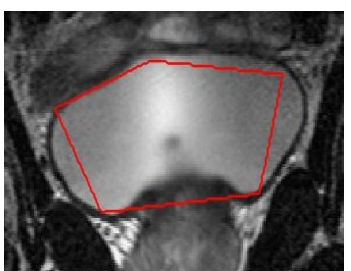

(a)

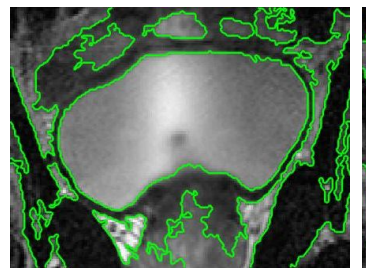

(b)

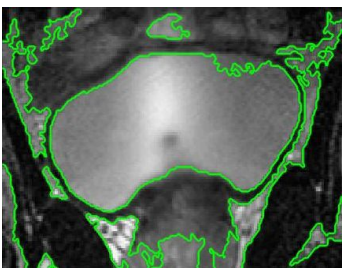

(c)

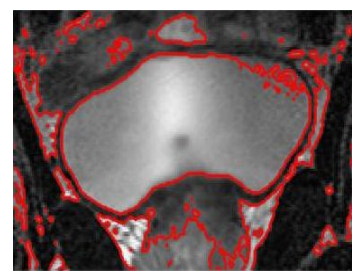

(d)

Fig. 11: Experiments on an MR image of bladder (downloaded from [12]): (a) Initial contour. Result of our model for: (b) $\sigma=8$. (c) $\sigma=30$. (d) Result of the ORACM model.

\section{Conclusion}

In this paper a region-based active contour method for image segmentation is presented which can efficiently segment the images with intensity inhomogeneity by exploiting the local image information. In particular, A new SPF function is constructed which uses local mean image information provided by the LBF model and helps to segment intensity inhomogeneous regions. Experimental results on both synthetic and real images demonstrated the advantages through accuracy, rapidity and insensitivity to the initial contour location of the proposed model over the related models.

\section{References}

[1] M. Kass, A. Witkin, and D. Terzopoulos, "Snakes: Active contour models," International Journal of Computer Vision, 1(4):321-331, 1987. DOI:10.1007/BF00133570

[2] V. Caselles, R. Kimmel, and G. Sapiro, "Geodesic active contours," International Journal of Computer Vision, 22(1):61-79, 1997. DOI:10.1023/A:1007979827043

[3] C. Li, C. Xu, C. Gui, and M. D. Fox, "Level set evolution without re-initialization: A new variational formulation", in Proc. IEEE Conf. Comput. Vis. Pattern Recognit., San Diego, 1:430-436, 2005. DOI: 10.1109/CVPR.2005.213 
[4] C. Li, C. Xu, C. Gui, and M. D. Fox, "Distance regularized level set evolution and its application to image segmentation," IEEE Trans. Image Process., 19(12):3243-3254, 2010. DOI: 10.1109/TIP.2010.2069690

[5] T. Chan and L. Vese, "Active contours without edges," IEEE Trans. Image Process., 10(2):266-277, 2001. DOI:10.1109/83.902291

[6] K. Zhang, L. Zhang, H. Song, and W. Zhou, "Active contours with selective local or global segmentation: A new formulation and level set method", Image and Vision Computing, 28(4):668676, 2010. DOI:10.1016/j.imavis.2009.10.009

[7] M. F. Talu, "ORACM: Online region-based active contour model," Expert Systems with Applications, 40(16):6233-6240, 2013. DOI:10.1016/j.eswa.2013.05.056

[8] N. M. Altarawneh, S. Luo, B. Regan, C. Sun, and F. Jia "Global Threshold and Region-based Active Contour Model for Accurate Image Segmentation," Signal and Image Processing, 5(3):1-11, 2014. DOI : $10.5121 /$ sipij.2014.530

[9] T.T. Tran, V.T. Pham, Y.J. Chiu, and K.K. Shyu, "Image Segmentation based on Geodesic aided Chan-Vese Model," in: Proc. of the 3rd IEEE International Conference on Computer Science and Information Technology, Chengdu, 1:315-317, 2010. DOI:10.1109/ICCSIT.2010.5563751

[10] D. Mumford and J. Shah, "Optimal approximations by piecewise smooth functions and associated variational problems," Commun. Pure Appl. Math., 42(5):577-685, 1989. DOI: $10.1002 /$ cpa.3160420503

[11] T. Liu, H. Xu, W. Jin, Z. Liu, Y. Zhao, and W. Tian, "Medical Image Segmentation Based on a Hybrid Region-Based Active Contour Model", Computational and Mathematical Methods in Medicine, 2014:10 pages,, Article ID 890725, 2014. DOI:10.1155/2014/890725

[12] C. Li, C. Kao, J. Gore, and Z. Ding, "Implicit Active Contours Driven by Local Binary Fitting Energy", in Proc. IEEE Conf. Computer Vision and Pattern Recognition, Minneapolis, 1-7, 2007. DOI:10.1109/CVPR.2007.383014

[13] X. Li, D. Jiang, Y. Shi, and W. Li, "Segmentation of MR image using local and global region based geodesic model", Biomedical engineering online, 14(1):p8, 2015. DOI:10.1186/1475-925X-14-8

[14] S. Liu, and Y. Peng, "A local region-based Chan-Vese model for image segmentation", Pattern Recognition, vol. 45, no. 7, pp 2769-2779, Jul. 2012. DOI:10.1016/j.patcog.2011.11.019

[15] F. Akram, J. H. Kim, H. U. Lim, and K. N. Choi, "Segmentation of Intensity Inhomogeneous Brain MR Image Using Active Contours", Computational and Mathematical Methods in Medicine, 2014: 14 pages, Article ID 194614, 2014. DOI:10.1155/2014/194614

[16] S. Osher, J. A. Sethian, "Fronts propagating with curvature-dependent speed: algorithms based on Hamilton-Jacobi formulations", J. Computational Physics, 79(1):12-49, 1988. DOI:10.1016/00219991(88)90002-2

[17] H. Xu, T. Liu, and G. Wang, "Hybrid geodesic region-based active contours for image segmentation", Computers \& Electrical Engineering, 40(3):858-869, 2014. DOI:10.1016/j.compeleceng.2013.07.026

[18] Y. Yang, and B. Wu, "Convex image segmentation model based on local and global intensity fitting energy and split Bregman method", Journal of Applied Mathematics, 2012:16 pages, Article ID 692589, 2012. DOI:10.1155/2012/692589

[19] K. Zhang, L. Zhang, K. M. Lam, and D. Zhang, "A Local Active Contour Model for Image Segmentation with Intensity Inhomogeneity ", arXiv preprint arXiv:1305.7053. 2013.

[20] C. Li, R. Huang, Z. Ding, J. C. Gatenby, D. N. Metaxas, and J. C. Gore, "A level set method for image segmentation in the presence of intensity inhomogeneities with application to MRI", IEEE Trans. Image Process., 20(7):2007-2016, 2011. DOI:10.1109/TIP.2011.2146190 